\title{
Artificial Intelligence and Learning Systems Methods in Supporting Long-Term Acoustic Climate Monitoring
}

\author{
M. KŁACZYŃSKI* AND T. WsZOŁEK \\ AGH - University of Science and Technology, Faculty of Mechanical Engineering and Robotics \\ Department of Mechanics and Vibroacoustics, al. A. Mickiewicza 30, 30-059 Krakow, Poland
}

\begin{abstract}
Developing effective methods for automatic identification of noise sources is currently one of the most important tasks in long-term acoustical climate monitoring of the environment. Manual verification of recorded data, when it comes to proper determination of noise levels, is time-consuming and costly. A possible solution is to use pattern recognition techniques for acoustic signal recorded by a monitoring station. This paper presents usefulness of special directed measurement techniques, acoustic signal processing, and classification methods using artificial intelligence (the Sammon mapping) and learning systems methods (Support Vector Machines) in the recognition of corona audible noise from ultra-high voltage AC transmission lines.
\end{abstract}

DOI: $10.12693 /$ APhysPolA.123.1024

PACS: 43.60.Lq, 07.05.Mh

\section{Introduction}

One of the adverse occurrences associated with transmission of electricity through ultra-high voltage AC transmission lines is corona which, among other features, is a source of acoustic signal. Such signal can be a valuable diagnostic symptom of a condition of certain elements of the transmission circuit, while, on the other hand, it is a source of noise, and residents in the immediate vicinity of such high voltage lines often complain about inconvenience associated with said noise. Large randomized factors stimulating corona phenomena, especially weather conditions and technical state of the conductors and insulators' surface, cause such generated acoustic signal having a random nature regarding both the level and shape of the spectrum [1].

The above-mentioned randomness causes great difficulty in accurate measurement of long-term noise levels deriving from corona. The indicators can be determined on the basis of short-term measurements in characteristic states of weather, time of day or seasons of the year, either from data gathered in long-term continuous monitoring systems. Manual verification of recorded data is time-consuming and costly. A possible solution is to use pattern recognition methods for identification of acoustic noise sources based on data recorded by a monitoring station $[2,3]$.

\section{Research methodology}

In this study, recordings from a long-term acoustic monitoring station were used for detection and identification of corona audible noise (CAN). The monitoring measurements were carried out in several locations

\footnotetext{
*corresponding author
}

near ultra-high voltage AC (UHV AC) transmission lines. This material was used in an earlier authors' study [4].

The monitoring station (Fig. 1) registered data by means of an all-weather microphone [5] in the mode of sound level continuous spectrum measurement in $1 / 3$ octave bands in the frequency range between $20 \mathrm{~Hz}$ and $20 \mathrm{kHz}$. Additionally, the A-, C-, and Z-weighted sound levels were registered. The averaged spectrum data was recorded to a buffer-type file every $10 \mathrm{~s}$, while other data, including $L_{\text {Aeq }}$ values and statistical levels of the $1 / 3$ octave spectrum and the A-, C-, and Z- weighted sound levels, were recorded every 15 minutes. In the same time step, the following average weather conditions were registered: temperature, atmospheric pressure, rainfall, humidity, and wind speed and direction. The registered data was used for extraction of the distinctive features of $\mathrm{CAN}$, and further for define the feature vector.

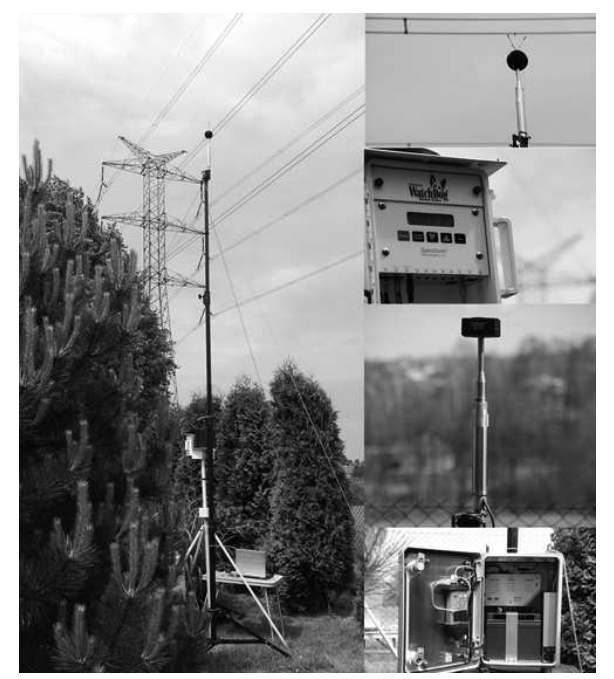

Fig. 1. The long-term acoustic monitoring station near $400 \mathrm{kV}$ transmission line. 
In the authors' previous papers $[1,4,6,7]$, several versions of the feature vector parameters were presented, and the proper selection of the feature space was extensively discussed. Finally, the space has been reduced from 19 to 10 elements [4] and contains:

- normalized (to $M_{0}$ ) spectral moments of 1 and 2 order $\left(M_{1}, M_{2}\right)$ for the whole frequency band $(20 \mathrm{~Hz}$ to $20 \mathrm{kHz}$ );

- coefficients of the signal power divided into the tonal components band from $20 \mathrm{~Hz}$ to $400 \mathrm{~Hz}\left(P C_{1}\right)$ and the noise components band from $1000 \mathrm{~Hz}$ to $10 \mathrm{kHz}\left(P C_{2}\right)$;

- coefficients useful in detection of the tonal components $100 \mathrm{~Hz}$ and $200 \mathrm{~Hz}$ in the spectra of registered samples $\left(T C_{100}, T C_{200}\right)$;

- $L_{\mathrm{A}} 90$ - the $L 90$ statistical level for A-weighted sound level (carrying the information concerning the interference level);

- $R F$ - rain fall $[\mathrm{mm} / \mathrm{h}], W S-$ wind speed $[\mathrm{m} / \mathrm{s}]$, $R H$ - relative humidity [\%].

The spectral moment of the $m$-th order is described by a general relation (1):

$$
M_{m}(t)=\sum_{i=0}^{N}\left|G_{n}\left(f_{i}\right)\right|\left[f_{i}\right]^{m},
$$

where $G_{n}(f)$ is the frequency spectrum of the $n$-th data record, $f_{i}$ - midpoint frequency for the $i$-th frequency band defined for the spectral analysis, $f_{0}$ - lower band frequency, $f_{N}-$ upper band frequency.

The spectral moment of the 0 -th order $M_{0}$ is used for normalization of the higher order moments and is given by the following relation:

$$
M_{0}(t)=\sum_{i=0}^{N}\left|G_{N}\left(f_{i}\right)\right| .
$$

It is usually convenient to use spectral moments normalized according to relation:

$$
M_{u}(m)=\frac{M(m)}{M(0)} .
$$

The $M_{1}$ moment (4) can be interpreted as a spectrum's center of gravity (weighted average frequency):

$$
M_{1}(t)=\frac{\sum_{i=0}^{N}\left|G_{N}\left(f_{i}\right)\right| \cdot f_{i}}{M_{0}(t)} .
$$

The $M_{2}$ moment (5) can be interpreted as the spectrum dispersion around the spectrum's center of gravity:

$$
M_{2}(t)=\sqrt{\frac{\sum_{i=0}^{N} G_{N}\left(f_{i}\right) \cdot f_{i}^{2}}{M_{0}(t)}-M_{1}^{2}(t) .}
$$

In addition to the spectral moments, the so-called "relative power coefficients" of the signal spectrum were determined. These parameters describe the ratio of the signal power in a selected frequency band to the signal power in the whole $20-20000 \mathrm{~Hz}$ band. The signal power coefficient of the $m$-th order can be determined by the formula:

$$
P C_{m}=\frac{\sum_{f=f_{d}}^{f_{g}} G_{N}(f)}{\sum_{f=20}^{20000} G_{N}(f)},
$$

where $\underline{f}_{\mathrm{d}}, \underline{f}_{\mathrm{g}}$ are the limits of the selected frequency band.

For the purpose of this research, two power coefficients have been used: $P C_{1}$ calculated from $20 \mathrm{~Hz}$ to $400 \mathrm{~Hz}$ frequency band (the band where rainfall noise effects and the tonal components of corona noise are encountered) and $P C_{2}$ calculated from $1 \mathrm{kHz}$ to $10 \mathrm{kHz}$ frequency band (the band where the noise components of corona process signal are located).

Tonal coefficients useful in detection of the tonal components are defined as

$$
T C_{f}=\left(\frac{\partial G_{N}(f)}{\partial f}\right),
$$

where $f$ is the frequency of a given tonal component, and $f=100 \mathrm{~Hz}, 200 \mathrm{~Hz}$.

Therefore, the space of the feature vector is based on the spectral coefficients, statistical data of the Aweighted sound level, and the meteorological data and it can be described symbolically as:

$$
\begin{aligned}
& X n=\left\langle M_{1}, M_{2}, P C_{1}, P C_{2}, T C_{100}, T C_{200}, R H, R F,\right. \\
& \left.W S, L_{\mathrm{A}} 90\right\rangle .
\end{aligned}
$$

\section{Experimental study}

\subsection{Research material}

The database used in the study contained 6371 records of the feature vector (8) with the imposed recognition qualification thresholds: " 0 " - no corona effect (6012 records); "0.5" - average corona effect signal (215 records); "1" - corona effect signal (144 records). This classification was proposed by subjective decision based on listening to audio recordings of the data.

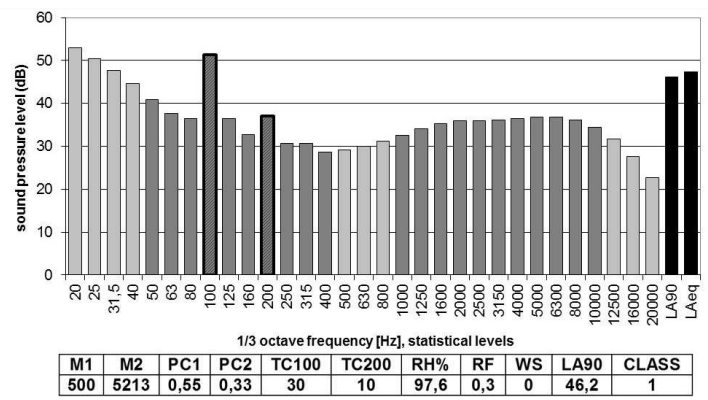

Fig. 2. Spectrum of intense corona effect.

Example spectra registered by the acoustic monitoring stations are shown in Figs. 2-4, together with respective 


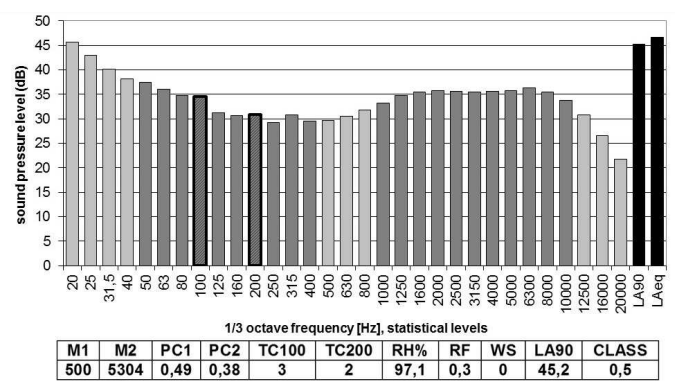

Fig. 3. Spectrum of average corona effect.

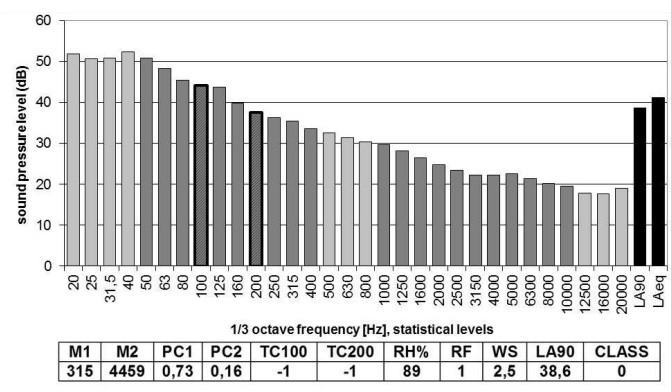

Fig. 4. Spectrum of no corona effect.

values of the feature vector and the corona noise classification. In the spectrum of the line audible noise, two characteristic elements can be distinguished: a broadband component - a noise in the range from about $1 \mathrm{kHz}$ to $10 \mathrm{kHz}$, described alternately as 'frying', 'crackle, 'wheezing' - and a pure-tone component at frequencies of $100 \mathrm{~Hz}$ (for a $50 \mathrm{~Hz}$ system) and multiples (e.g. $200 \mathrm{~Hz}$ ). The pure tones are superimposed on broadband noise. A 'hum' of the second harmonic (hum noise) is at the same time considered as the most perceptible.

\subsection{The Sammon mapping}

The feature vectors represent recorded data in a 10dimensional space. This multidimensional structure is difficult to interpret by visual inspection. Therefore, it is important to develop an understandable and perceptible graphical illustration of such set of data. In certain studies [8,9], solving this problem by applying the Sammon representation [10] is proposed. The Sammon representation allows presenting multi-variant data on a plane. Transformation is based upon selection of twodimensional vectors created in the way which minimizes the error function of the distance between the considered objects.

The error function is defined according to expression:

$$
E=\frac{1}{\sum_{i<j} d_{i j}^{*}} \sum_{i<j}^{n} \frac{\left(d_{i j}^{*}-d_{i j}\right)^{2}}{d_{i j}^{*}},
$$

where $d_{i j}^{*}=d\left(x_{i}, x_{i}\right)$ is the distance between $i$-th and $j$ th object in the original space, and $d_{i j}=d\left(y_{i}, y_{i}\right)$ is the distance between $i$-th and $j$-th object in 2-dimensional space. The distance is calculated using Euclidean Metric.
The task of the mapping consists in selection of a vector $y$ minimizing the error function. To this end, Newton's optimization method [11] was used simplified to the form

$$
y_{p q}(t+1)=y_{p q}(t)-\eta \Delta_{p q}(t)
$$

where

$$
\begin{aligned}
& \Delta_{p q}(t)=\frac{\frac{\partial E}{\partial y_{p q}}}{\left|\frac{\partial^{2} E}{\partial y_{p q}^{2}}\right|}, \\
& \frac{\partial E}{\partial y_{p q}}=-\frac{2}{c} \sum_{j=1, j \neq p}^{n}\left[\frac{d_{p j}^{*}-d_{p j}}{d_{p j}^{*} \cdot d_{p j}}\right]\left[y_{p q}-y_{j q}\right], \\
& \frac{\partial^{2} E}{\partial y_{p q}^{2}}=-\frac{2}{c} \sum_{j=1, j \neq p}^{n} \frac{1}{d_{p j}^{*} d_{p j}}\left[\left(d_{p j}^{*}-d_{p j}\right)\right. \\
& \left.-\frac{\left(y_{p q}-y_{j q}\right)^{2}}{d_{p j}} \cdot\left(1+\frac{d_{p j}^{*}-d_{p i}}{d_{p i}}\right)\right] .
\end{aligned}
$$

Example results of the Sammon representation for the examined group of corona audible noise are presented in Figs. 5 and 6. Separation of the objects related to "1" (corona effect) and " 0 " (no corona effect) is clearly seen in Fig. 5 and confirms that the proposed 10-parameters feature space can be considered useful for identification purposes. However, separation of the objects related to " 0.5 " (average corona effect) and " 0 " (no corona effect) is fuzzy (cf. Fig. 6).

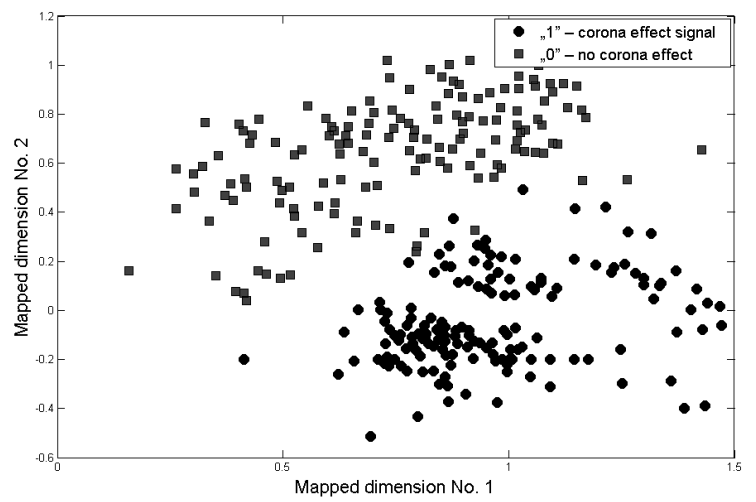

Fig. 5. Visualization of 10-dimensional feature vectors with Sammon's representation ("1" - corona effect signal, " 0 " - no corona effect).

This is related to the problem that often the medium and low corona noise is masked by background noise; therefore, despite assigning the object (record) to the class " 0.5 ", it is in cluster of the class " 0 ".

To evaluate the mapping from 10-dimensional to 2dimensional space, the modified the Sebestyen criterion [12] was used. This criterion is based on the degree of dispersion intra-classes and global dispersion inter-classes. The results of Sebestyen criterion applied to data before and after the Sammon mapping are shown in Tables I and II. The Sammon mapping from multi-dimensional 


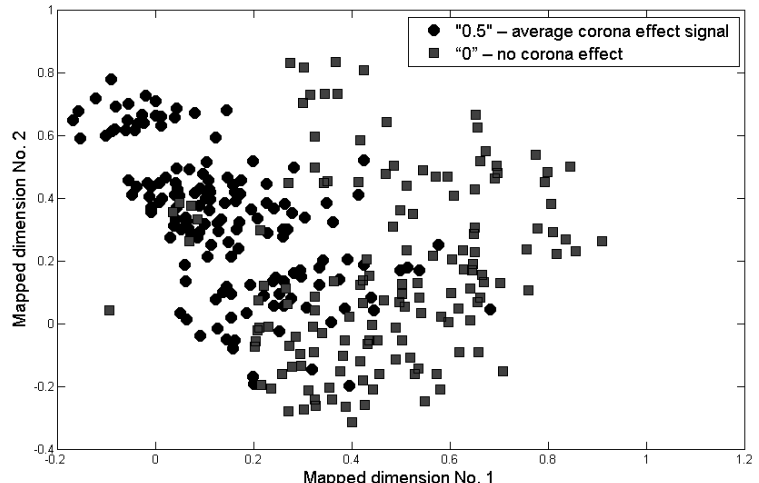

Fig. 6. Visualization of 10-dimensional feature vectors with Sammon's representation ("0.5" - average corona effect signal, "0" - no corona effect).

space onto a plane is not a real reflection of the original data set. However, it retains features such as inter-class and intra-class dispersion in a fairly high compliance with the original vectors.

TABLE I

Summary of the Sebestyen criterion for data before and after the Sammon mapping for classes: "1" — corona effect, "0" — no corona effect.

\begin{tabular}{|c|c|c|}
\hline & Actual data & Data after mapping \\
\hline $\begin{array}{ll}\text { Inter-classes } & \text { dispersion } \\
\text { measure } & \end{array}$ & 0.64 & 0.67 \\
\hline $\begin{array}{l}\text { Intra-classes dispersion } \\
\text { measure }\end{array}$ & 0.24 & 0.22 \\
\hline $\begin{array}{l}\text { Quantitative assessment } \\
\text { of classes separation }\end{array}$ & 1.41 & 1.59 \\
\hline
\end{tabular}

TABLE II

Summary of the Sebestyen criterion for data before and after the Sammon mapping for classes: " 0.5 " - average corona effect, "0" — no corona effect.

\begin{tabular}{|c|c|c|}
\hline & Actual data & Data after mapping \\
\hline $\begin{array}{l}\text { Inter-classes dispersion } \\
\text { measure }\end{array}$ & 0.32 & 0.32 \\
\hline $\begin{array}{l}\text { Intra-classes dispersion } \\
\text { measure }\end{array}$ & 0.20 & 0.19 \\
\hline $\begin{array}{l}\text { Quantitative assessment } \\
\text { of classes separation }\end{array}$ & 0.66 & 0.77 \\
\hline
\end{tabular}

\subsection{Support Vector Machines classification}

Support Vector Machines (SVM) consist of a group of supervised learning methods that can be applied to twogroup classification problems. The classification tasks are performed by constructing hyperplanes in a multidimensional space, which separates objects with different class labels. SVM algorithm based on linear classifier was proposed by Vapnik in 1963 [13]. However, this method has been extended to nonlinear classifiers by applying the kernel function, which allows the algorithm to fit the maximum-margin hyperplane in a transformed feature space [14]. SVM are still being improved [15]. In the present study a polynomial kernel function was used of the order chosen experimentally (in the range from 3-rd to 20-th) to execute a line separating. Criterion has been accepted as the minimum number of support vectors. Example results of corona audible noise classification are presented in Figs. 7-10.

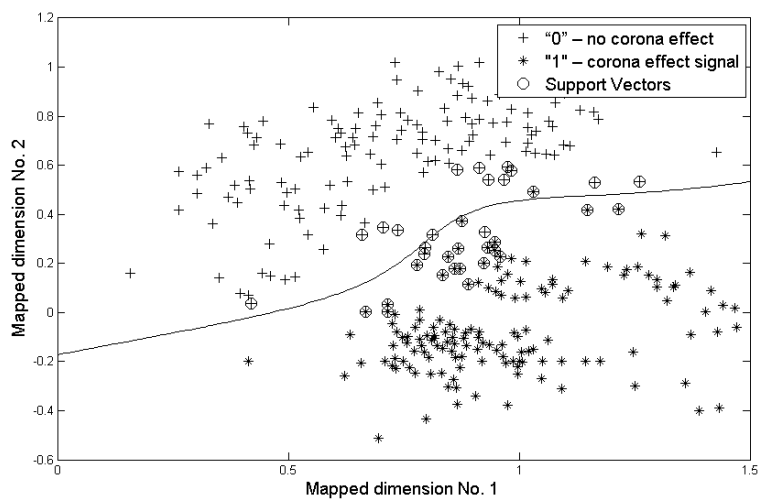

Fig. 7. Visualization of SVM classification method, 3rd order polynomial line separating.

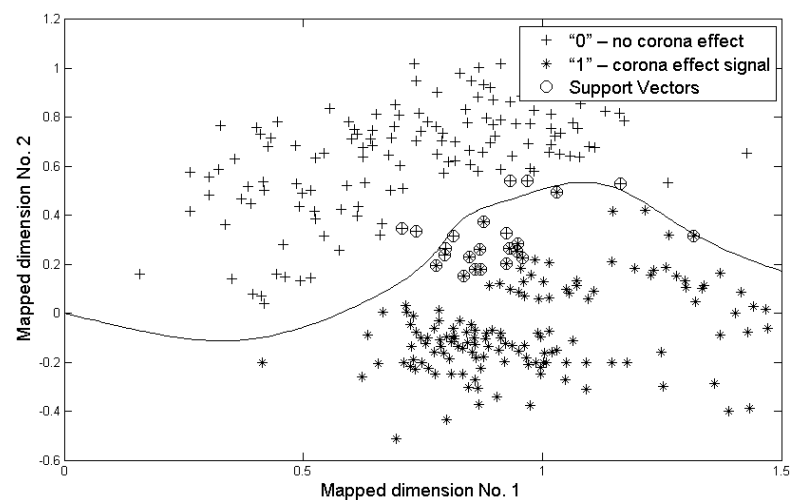

Fig. 8. Visualization of SVM classification method, 7th order polynomial line separating.

Classification of the objects related to " 1 " (corona effect) and " 0 " (no corona effect) is very good for the order 7-th and higher of polynomial kernel function (see Fig. 8). For lower order of this function, greater amount of support vectors is necessary (see Fig. 7), but classification is still possible on high level.

Because the objects related to " 0.5 " (average corona effect) and " 0 " (no corona) are fuzzy, the classification is possible with 20-th order polynomial kernel function (see Fig. 10). 


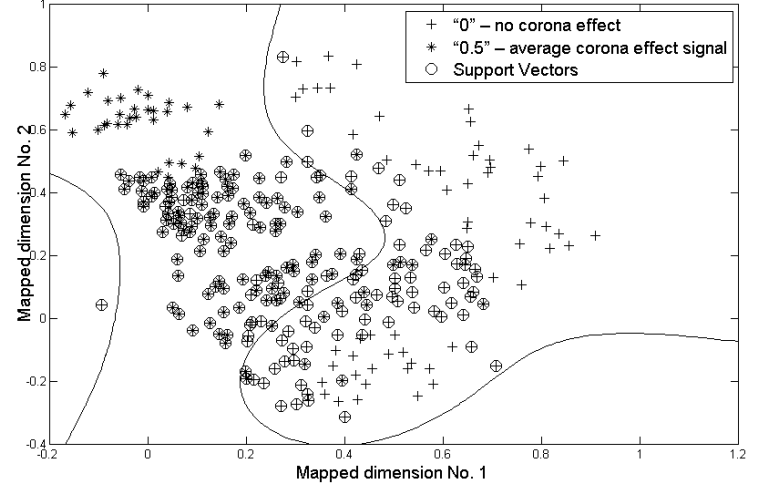

Fig. 9. Visualization of SVM classification method, 4th order polynomial line separating.

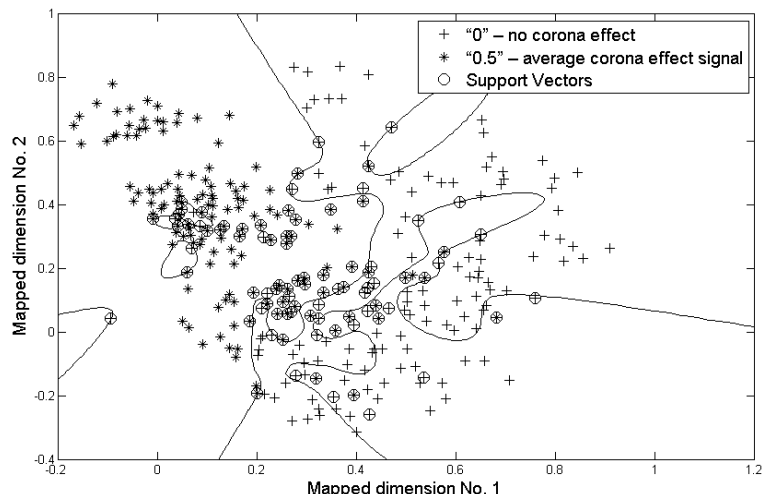

Fig. 10. Visualization of SVM classification method, 20 -th order polynomial line separating.

\section{Conclusions}

This paper presents a method for automatic classification of measured data collected in a long-term acoustic monitoring station of corona noise generated by ultrahigh voltage transmission lines. Classification of registered samples was carried out using the Support Vector Machines technique. The proposed feature vector consists of 10 features, representing parameters of the corona audible noise spectral density distribution - spectral mo- ments, spectral power coefficients, tonal components contribution coefficients, and meteorological data (humidity, rainfall, wind speed). The developed algorithm can be incorporated into existing monitoring systems of acoustic climate, and also used in the analysis of recordings from existing systems.

\section{Acknowledgments}

The paper has been written and the respective research work undertaken within the project 2011/01/D/ST6/07178 (National Science Centre).

\section{References}

[1] Z. Engel, T. Wszołek, Applied Acoustics 47, 149 (1996).

[2] M. Kłaczyński, T. Wszołek, Acta Phys. Pol. A 121, A-179 (2012).

[3] D. Dąbrowski, E. Jamro, W. Cioch, Acta Phys. Pol. A 118, 41 (2010).

[4] T. Wszołek, M. Kłaczyński, Archiv. Acoust., (in press).

[5] G. Wszołek, Acta Phys. Pol. A 119, 6-A (2011).

[6] T. Wszołek, Archiv. Acoust. 34, 1 (2009).

[7] T. Wszołek, R. Tadeusiewicz, in: 50th Open Seminar of Acoustics, Szczyrk-Gliwice, 2003, p. 512.

[8] W. Cioch, Machine Dynam. Probl. 27, 3 (2003).

[9] Z. Engel, M. Kłaczyński, W. Wszołek, Int. J. Occupational Safety Ergon. (JOSE) 13, 4 (2007).

[10] J. Sammon, in: IEEE Trans. Computers 18, (1969).

[11] R. Fletcher, Practical methods of optimization, 2nd ed., John Wiley, New York 1987, p. 44.

[12] W. Sobczak, W. Malina, Methods of Selection and Reduction of Information, WNT, Warszawa 1985, p. 199, (in Polish).

[13] V. Vapnik, A. Lerner, in: Automation and Remote Control 24, (1963).

[14] B. Boser, I. M. Guyon, V. Vapnik, in: Proceedings of the Fifth Annual Workshop on Computational Learning Theory, ACM Press, New York 1992.

[15] I. Steinwart, A. Christmann, Support Vector Machines, Springer-Verlag, New York 2008, p. 287. 